\title{
ARK5 promotes invasion and migration in hepatocellular carcinoma cells by regulating epithelial-mesenchymal transition
}

\author{
ZHIYU YE, XUDONG CHEN and XIAOGANG CHEN \\ Department of Hernia and Hepatobiliary Surgery, Ningbo First Hospital, Ningbo, Zhejiang 315000, P.R. China
}

Received July 20,2016; Accepted October 24, 2017

DOI: $10.3892 / \mathrm{ol} .2017 .7453$

\begin{abstract}
Hepatocellular carcinoma (HCC) is the third leading cause of cancer-associated mortality worldwide. The highly invasive nature of HCC leads to poor prognosis in patients with malignant HCC. AMPK-related protein kinase 5 (ARK5) is a key mediator of migratory activity in human cancer cells. However, the role of ARK5 in invasion and metastasis of HCC cells remains unclear. The present study attempted to determine whether ARK5 is involved in invasion and migration via regulation of epithelial-mesenchymal transition (EMT). Wound healing and Transwell Matrigel invasion assays were utilized to detect the ability of the epithelial Huh7 and mesenchymal SNU387 HCC cells to migrate and invade. Next, the expression of ARK5 and EMT markers, E-cadherin and vimentin, were examined by western blot analysis. Inhibition of ARK5 was able to significantly reduce the ability HCC cells to invade and metastasize. Furthermore, the knockdown of ARK5 was able to reverse the process of EMT in HCC cells. These data suggested that ARK5 may serve an important role in regulating EMT in HCC cells. Taken together, these findings indicate that ARK5 is a potential molecular target for the development of novel HCC therapeutics, which focus on cell invasion and EMT regulation.
\end{abstract}

\section{Introduction}

Hepatocellular carcinoma (HCC) is a malignancy with one of the highest mortality rates, and it has been ranked as the fifth most common malignancy globally (1). Although substantial progress has been made in the diagnosis and treatment of HCC, it remains the third leading cause of cancer-associated mortality globally (2). Therefore, there is an urgent requirement to investigate the molecular mechanisms by which HCC

Correspondence to: Dr Xiaogang Chen, Department of Hernia and Hepatobiliary Surgery, Ningbo First Hospital, 59 Liuting Street, Ningbo, Zhejiang 315000, P.R. China

E-mail: chenxiaogang1123@163.com

Key words: hepatocellular carcinoma, AMPK-related protein kinase 5, invasion and migration, epithelial-mesenchymal transition progresses and to find novel diagnostic factors and effective therapeutic strategies to improve patient survival rates.

The epithelial-mesenchymal transition (EMT) comprises a complex series of reversible events that may lead to the loss of epithelial cell adhesion and the indication of a mesenchymal phenotype (3). EMT serves crucial roles during embryonic development, tumor metastasis and invasion, and is one of the major molecular mechanisms through which invasion and metastasis are promoted during the oncogenic process $(4,5)$. Previous studies demonstrated that EMT activation in cancer cells contributed to tumor invasion and metastasis in various types of cancer, including HCC, resulting in aggressive cancer progression $(6,7)$. Taken together, these findings indicate that EMT is associated with metastasis.

AMPK-related protein kinase 5 (ARK5) is a member of the human AMP-activated protein kinase family. ARK5 was identified as a key molecule in mediating the migration of cancer cells in human pancreatic cancer cells, and its activation was induced by Akt-dependent Ser600 phosphorylation (8). It has been reported that the overexpression of ARK5 was involved in tumor progression and metastatic activity in colorectal cancer (9). Tumor malignancy, including invasion and metastasis, is accelerated by the activation of Akt, a process that has been demonstrated for breast, ovarian, colorectal and pancreatic cancer, and squamous cell carcinoma (10-13). ARK5 was reported to promote invasion and metastasis in cancer cells, including breast cancer, colorectal cancer and glioma $(9,14,15)$. However, the mechanism by which ARK5 alters invasion and metastasis has not been fully identified in HCC cells.

In the present study, the authors hypothesized that ARK5 may be involved in HCC cell invasion and metastasis through EMT. Suppression of ARK5 may be used as a potential target for the treatment of HCC. To investigate this hypothesis, the role of ARK5 in invasion and metastasis of cancer was investigated using HCC cells.

\section{Materials and methods}

Cell culture. The human HCC Huh7 and SNU387 cells were purchased from the American Type Culture Collection (Manassas, VA, USA). Huh7 cells were cultured in Dulbecco's Modified Eagle's Medium (DMEM; Gibco; Thermo Fisher Scientific, Inc., Waltham, MA, USA) containing $10 \%$ fetal bovine serum (FBS; Gibco; Thermo Fisher Scientific, Inc.) and 
$1 \%$ penicillin/streptomycin (Sigma-Aldrich; Merck KGaA, Darmstadt, Germany). SNU387 cells were maintained in RPMI-1640 medium (Gibco; Thermo Fisher Scientific, Inc.) supplemented with $10 \%$ FBS and $1 \%$ penicillin/streptomycin. All cells were grown in a humidified incubator at $37^{\circ} \mathrm{C}$ and with $5 \% \mathrm{CO}_{2}$.

Short interfering RNA (siRNA) transfection. HCC Huh7 and SNU387 cells were transfected with $100 \mathrm{nmol} / \mathrm{l}$ ARK5 siRNA (Santa Cruz Biotechnology, Inc., Dallas, TX, USA) or negative siRNA control (Invitrogen; Thermo Fisher Scientific, Inc.) using Lipofectamine ${ }^{\circledR} 2000$ (Invitrogen; Thermo Fisher Scientific, Inc.) following the manufacturer's protocol. The transfection medium Opti-MEM (Gibco; Thermo Fisher Scientific, Inc.) was used. After $6 \mathrm{~h}$, the transfection medium was removed, and the cells were maintained in DMEM and RPMI-1640 medium for an additional $24 \mathrm{~h}$, at which point all subsequent experiments were performed.

Western blot analysis. HCC Huh7 and SNU387 cells were washed with cold phosphate-buffered saline (PBS) and treated with cell lysis buffer (Cell Signaling Technology, Inc., Danvers, MA, USA) at $4^{\circ} \mathrm{C}$ or on ice for $2 \mathrm{~h}$. The concentration of the proteins was measured using BCA Protein Assay kit (Pierce; Thermo Fisher Scientific, Inc.). The protein (40 $\mu \mathrm{g}$ per lane) were separated by $10 \%$ SDS-PAGE and transferred onto polyvinylidene difluoride membranes (EMD Millipore, Billerica, MA, USA). The membranes were then blocked with $5 \%$ bovine serum albumin (Sangon Biotech Co., Ltd., Shanghai, China) in $0.1 \%$ Tween-20 (TBS/T) and incubated with primary antibodies against ARK5 (cat no. ab71814), E-cadherin (cat no. ab76055) and vimentin (cat no. ab8978) (all 1:1,000; Abcam, Cambridge, MA, USA) at $4^{\circ} \mathrm{C}$ overnight. The membranes were then washed three times with TBST and then incubated with the appropriate secondary antibody (1:2,000, Santa Cruz Biotechnology, Inc., Dallas, TX, USA) at $37^{\circ} \mathrm{C}$ for $2 \mathrm{~h}$. The protein bands were visualized by chemiluminesence using an enhanced chemiluminesence kit (GE Healthcare, Piscataway, NJ, USA) and were quantified by densitometry using Image Lab 5.0 software (Bio-Rad Laboratories, Inc., Hercules, CA, USA). GAPDH (1:2,000; Cell Signaling Technology, Inc.; cat no. 5174) was used as an internal control.

Reverse transcription-quantitative polymerase chain reaction (RT-qPCR) analysis. Total RNA was extracted from HCC Huh7 and SNU387 cells with TRIzol (Invitrogen, Carlsbad, CA, USA). cDNA was synthesized using M-MLV Reverse Transcript reagent (Invitrogen; Thermo Fisher Scientific, Inc.) according to the manufacturer's protocol.qPCR was performed using SYBR Green PCR kit (Takara Bio, Inc., Otsu, Japan) and analyzed using an ABI Prism 7500 Real-time PCR system (Applied Biosystems, CA, USA) under the following reaction conditions: $50^{\circ} \mathrm{C}$ for $2 \mathrm{~min} ; 95^{\circ} \mathrm{C}$ for $30 \mathrm{sec}$ and followed by 40 cycles of $60^{\circ} \mathrm{C}$ for $30 \mathrm{sec}$. All reactions were performed in triplicate. ARK5 mRNA expression was quantified and reported as $2^{-\triangle \Delta \mathrm{Cq}}(16)$. The expression level of each gene was normalized to the expression level of GAPDH. The primers were as follows: ARK forward, 5'-GAGTCCACTCTATGC ATC-3' and reverse, 5'-GGCCACTATTGAGGACA-3'.

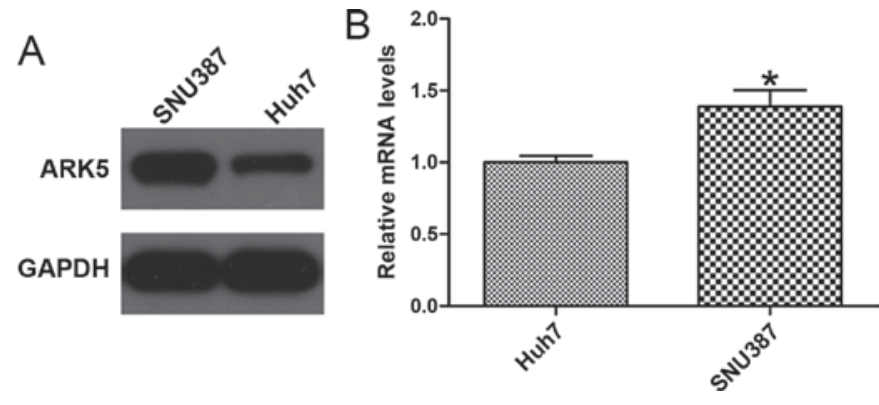

Figure 1. Expression of ARK5 in HCC cell lines. (A) Western blot analysis was used to examine the expression of ARK5 in HCC cells. Relative protein expression in HCC cells was quantified by evaluating the band density, and GADPH served as the control. All experiments were performed at least three times. (B) Relative expression of ARK5 was detected by reverse transcription-quantitative polymerase chain reaction in HCC cells. " $\mathrm{P}<0.05$ vs. Huh7 cells. ARK5, AMPK-related protein kinase 5; HCC, hepatocellular carcinoma.

Wound-healing assay. HCC Huh7 and SNU387 cells were seeded into 6-well plates at a density of $3 \times 10^{5}$ cells/well and cultured in the appropriate medium (DMEM used for Huh7 cells, and RPMI-1640 used for SNU387 cells) containing 10\% FBS, $1 \%$ penicillin/streptomycin at $37^{\circ} \mathrm{C}$ with $5 \% \mathrm{CO}_{2}$ for $24 \mathrm{~h}$ until $90 \%$ confluence, and then the medium was changed to the corresponding medium (DMEM used for Huh7 cells, and RPMI-1640 used for SNU387 cells) containing 0.05\% FBS, $1 \%$ penicillin/streptomycin overnight to synchronize the cells. The cells were wounded with $100 \mu \mathrm{l}$ pipette tips, and the cell debris was washed away with PBS. The wound scars were photographed with an inverted light microscope at 0 and $24 \mathrm{~h}$ after the scratch was made. The ratio of the remaining wound area relative to the initial wound area was calculated, and the wound area was quantified using Image-Pro Plus software (version 6.0; Media Cybernetics, Inc., Rockville, MD, USA).

Transwell Matrigel invasion assay. The Huh7 and SNU387 cells were seeded at a density of $5 \times 10^{4}$ cells/well in the upper chamber of a Transwell 24-insert plate with corresponding medium (DMEM used for Huh7 cells, and RPMI-1640 used for SNU387 cells). Following transfection with ARK5 siRNA $(100 \mathrm{nmol} / \mathrm{ml})$ for $6 \mathrm{~h}$, the cells were treated with TGF- $\beta 1$ $(10 \mathrm{ng} / \mathrm{ml})$ for $48 \mathrm{~h}$. The upper chambers were coated with Matrigel (BD Biosciences, San Jose, CA, USA), and the lower chamber contained the same medium supplemented with $10 \%$ FBS. After $24 \mathrm{~h}$, the bottom of the inserts were fixed in methanol for $10 \mathrm{~min}$ and stained with $\mathrm{H} \& \mathrm{E}$ for $3 \mathrm{~min}$ at $37^{\circ} \mathrm{C}$. The cells that had invaded to the lower surface were counted using an inverted phase-contrast microscope (magnification, 40x) and images were captured.

Statistical analysis. All data are expressed as the mean \pm standard deviation. Statistical analysis was performed using one-way analysis of variance (ANOVA) followed by Tukey's post hoc test. Student's t-test was also used. GraphPad Prism (version 5.0; GraphPad Software, Inc., La Jolla, CA, USA) was used to perform all statistical analysis. $\mathrm{P}<0.05$ was considered to indicate a statistically significant difference. All experiments were performed at least three times as independent experiments. 


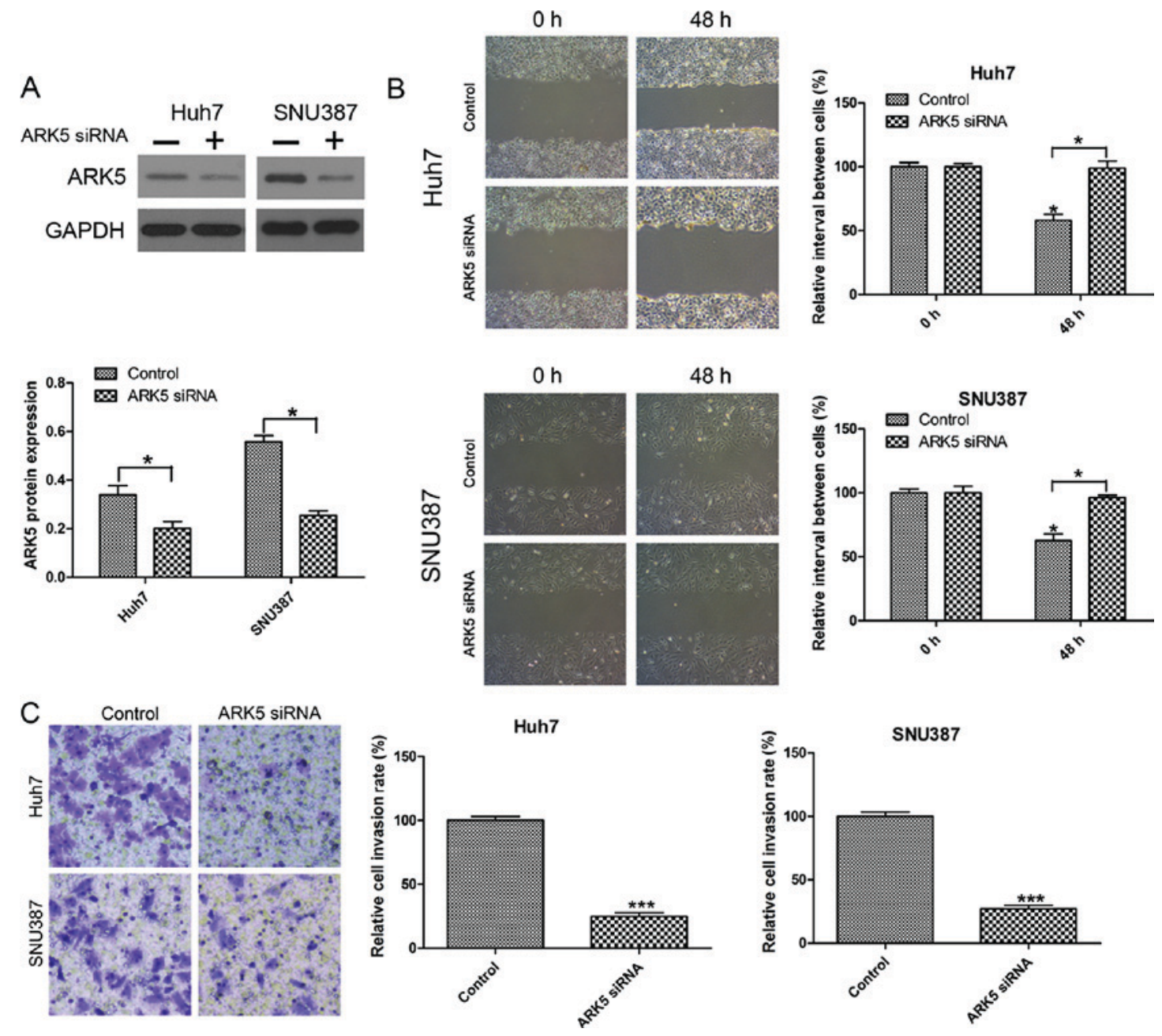

Figure 2. Knockdown of ARK5 inhibits invasion and migration in Huh7 and SNU387 cells. (A) Western blot was used to detect the expression of ARK5 in hepatocellular carcinoma cells that were transfected with ARK5 siRNA or negative siRNA control. GAPDH was used as the internal reference. (B) Wound healing assays indicate decreased motility in ARK5-knocked down Huh7 and SNU387 cells compared with the control. Magnification x200. "P<0.05 (C) Transwell migration assays. Images and quantification of migration of Huh7 and SNU387 cells in transfected with or without ARK5 siRNA. Migrated cells were stained with crystal violet and counted. Magnification $\times 200 .{ }^{* * *} \mathrm{P}<0.001$ vs. control; ARK5, AMPK-related protein kinase 5; siRNA, small interfering RNA.

\section{Results}

ARK5 expression level is different between Huh7 and SNU387 cell lines. To confirm whether the expression level of ARK5 was associated with HCC cell lines, western blotting and RT-qPCR were used to analyze the expression of ARK5. The HCC Huh7 cell line, with an epithelial phenotype, exhibited low expression of ARK5, whereas the mesenchymal phenotype HCC SNU387 cell line exhibited high expression of ARK5 (Fig. 1), indicating that the level of ARK5 may be associated with invasion and migration in HCC cells $(\mathrm{P}<0.05$ vs. Huh7).

Knockdown of ARK5 inhibits invasion and migration in Huh7 and SNU387 cells. To investigate the function of ARK5 in invasion and migration in HCC cells, the effect of ARK5 knockdown on metastasis was investigated by Transwell invasion and wound healing migration assays. The knockdown efficiency was examined by western blot analysis (Fig. 2A). The results indicated that inhibiting the expression of ARK5 was able to significantly reduce cell migration compared with the control under normal conditions after $24 \mathrm{~h}$ in wound healing migration assays (Fig. 2B).

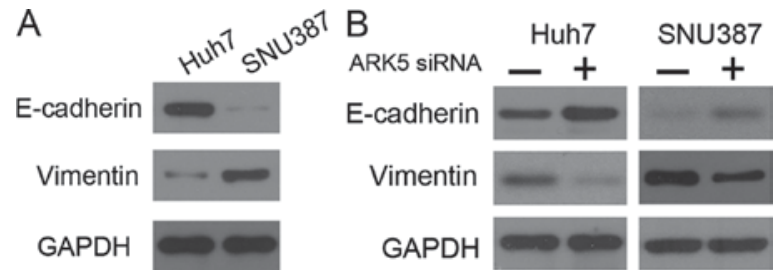

Figure 3. Knockdown of ARK5 upregulates the expression of E-cadherin and downregulates vimentin expression in hepatocellular carcinoma cells. (A) The expression of E-cadherin and vimentin in Huh7 and SNU387 cells. (B) Western blot analysis was used to detect the expression of ARK5, E-cadherin and vimentin following treatment with ARK5 siRNA or negative control siRNA. siRNA, small interfering RNA; ARK5, AMPK-related protein kinase 5.

In the Transwell invasion assay, it was observed that the number of HCC cells transfected with ARK5 siRNA passed through the Transwell membranes were decreased compared with the control group (Fig. 2C).

Knockdown of ARK5 may upregulate the expression of E-cadherin and downregulate vimentin expression in HCC cells. It has been reported that EMT serves a notable role in 
A
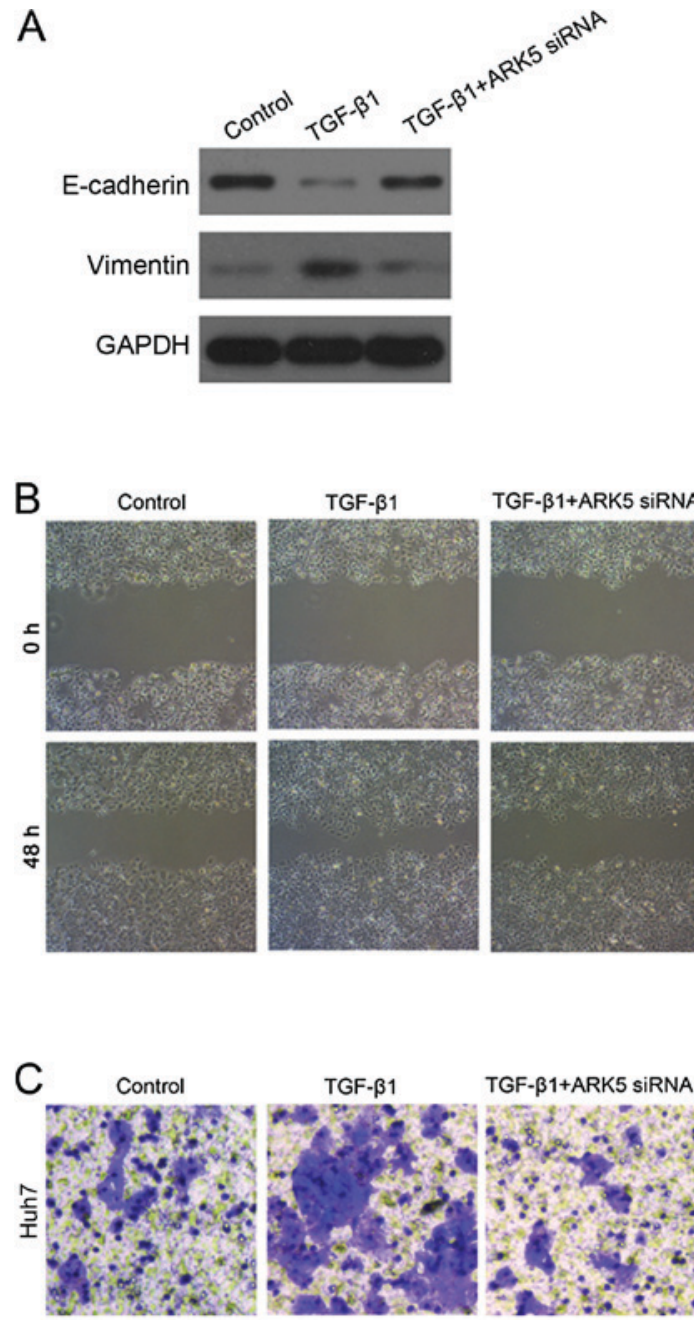
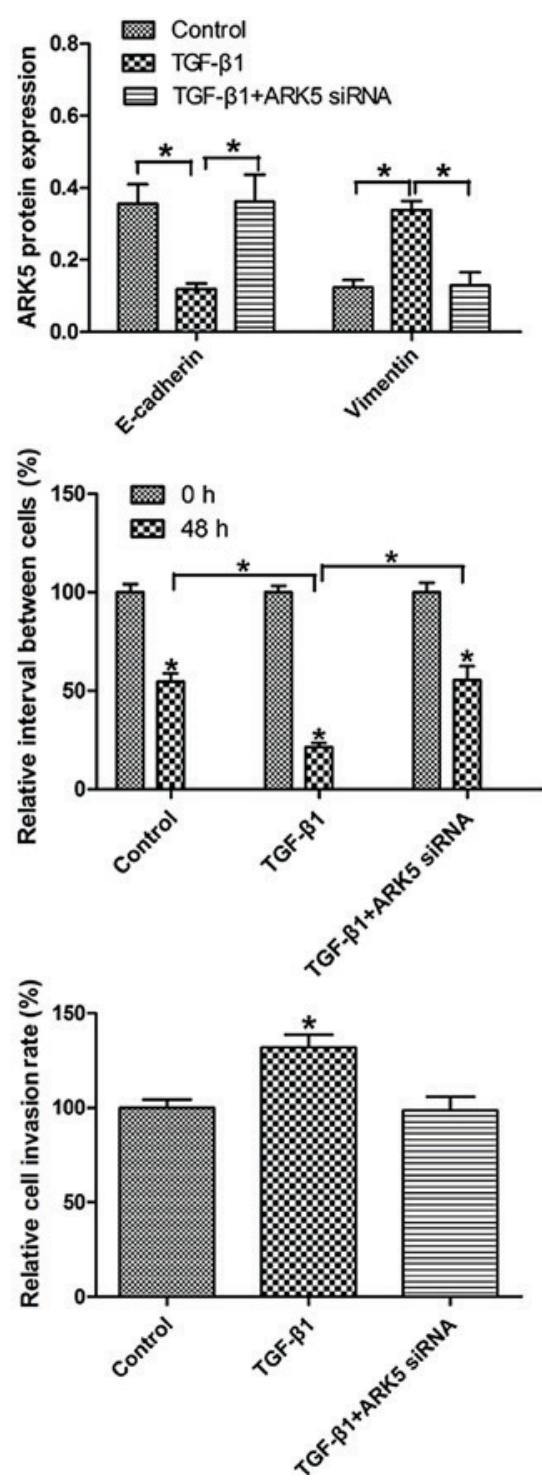

Figure 4. Inhibition of ARK5 reverses EMT under TGF- $\beta 1$ treatment in Hep3B cells. (A) Western blot analysis indicated that the knockdown of ARK5 in TGF- $\beta 1$-treated cells was able to reverse EMT, as demonstrated by increased E-cadherin expression and reduced vimentin expression. (B) Wound healing assays indicate that there was a significant difference between ARK5-siRNA +TGF- $\beta 1$ group and control in Huh7 cells. (C) Images and quantification of migration of Huh7 cells in TGF- $\beta 1$-treated cells which were transfected with or without ARK5 siRNA and control cells. Migrated cells were stained with crystal violet and counted. ${ }^{*} \mathrm{P}<0.05$ vs. the control. EMT, epithelial-mesenchymal transition; ARK5, AMPK-related protein kinase 5; TGF- $\beta 1$, transforming growth factor- $\beta 1$.

the development of drug resistance in a number of different tumor types, including cancer of the breast, lung, colon, and pancreas, with tumor cells that have gained drug resistance displaying a higher degree of malignant, and migratory and invasive ability (17-20). Studies have demonstrated that the inhibition of ARK5 results in the upregulation of E-cadherin expression and downregulation of vimentin expression in HCC, ovarian cancer and non-small lung cancer cells (21-23). In the present study, western blot analysis revealed that Huh7 cells exhibited high E-cadherin expression and low vimentin expression, whereas SNU387 cells exhibited the opposite pattern of expression (Fig. 3A). The results of the present study also demonstrated that siRNA-mediated silencing of ARK5 resulted in upregulation of E-cadherin and downregulation of vimentin in Huh7 and SNU387 cells (Fig. 3B). These results demonstrated that the inhibition of ARK5 may reverse EMT.
Inhibition of ARK5 reverses EMT under TGF- $\beta 1$ treatment in Hep $3 B$ cells. Previously, TGF- $\beta 1$ has been identified as the key driver in inducing EMT (24). Following exposure to $10 \mathrm{ng} / \mathrm{ml}$ TGF- $\beta 1$ for 48 h, E-cadherin expression in Huh7 cells was markedly decreased. Notably, the expression of E-cadherin in Huh7 cells was upregulated upon transfection with ARK5 siRNA following TGF- $\beta 1$ treatment compared with TGF- $\beta 1$-treated cells (Fig. 4A). Wound healing assays revealed that the knockdown of ARK5 was able to reverse TGF- $\beta 1$-induced cell migratory ability (Fig. 4B). Transwell invasion assays also revealed that the knockdown of ARK5 was able to reverse TGF- $\beta 1$-induced invasion (Fig. 4C). There was no significant difference between the group transfected with AKR5 siRNA combined with TGF- $\beta 1$ and the control in Huh7 cells. These findings indicated that the knockdown of ARK5 may reverse TGF- $\beta 1$-induced EMT and reduce the invasive and metastatic capabilities of Huh7 cells, which have an epithelial phenotype. 


\section{Discussion}

There is a high mortality associated with HCC, the fifth most common tumor worldwide, due to a lack of effective treatments (25). HCC is frequently diagnosed at an advanced stage and thus is associated with poor prognosis (26). It is known that cell invasion is essential for tumor metastasis, and Akt has been identified to serve a key role in invasion and metastasis of tumor cells (27-29). Therefore, it is important to understand the molecular basis of the involvement of ARK5 in the invasion and metastasis of HCC cell lines.

Evidence indicates that EMT serves a key role in carcinogenicity, metastasis, progression and acquired chemoresistance in many types of cancer, including in $\operatorname{HCC}(30,31)$. During EMT, expression of the epithelial marker E-cadherin decreases, whereas that of the mesenchymal marker vimentin increases (32). ARK5 is a tumor invasion-associated factor that is downstream of Akt signaling. Cancer cells with high ARK5 expression exhibit high invasive activity (11). It has been demonstrated that the inhibition of ARK5 is able to enhance drug sensitivity in hepatocellular carcinoma through EMT (21). However, prior to the present study, there were a limited number of reports concerning the role of ARK5 in the metastasis of other cancer types $(14,33)$, including $\mathrm{HCC}$.

The results of the present study indicated that the knockdown of ARK5 markedly decreased migration and invasion of HCC cells. Furthermore, the inhibition of ARK5 was also able to increase the expression of the epithelial marker E-cadherin and reduce expression of the mesenchymal marker vimentin in Huh7 and SNU387 cells, compared with the control.

TGF- $\beta 1$ is a pleiotropic cytokine that regulates cell proliferation and differentiation (34). TGF- $\beta 1$ induces a mesenchymal phenotype and functions as a tumor suppressor that restricts cell growth, resulting in the inhibition of cancer progression during the early stages of carcinogenesis (35). However, as cancer progresses, TGF- $\beta 1$ becomes a tumor promoter, performing dual roles in the progression and metastasis of HCC (36-38). The data reported in the present study indicated that TGF- $\beta$ treatment of HCC cells was able to induce invasion and migration. When the epithelial Huh7 cells were treated with TGF- $\beta 1$, the invasive capability and migratory rate of these cells was increased compared with the control. Notably, this increased invasive capability and migratory rate was reversed upon ARK5 knockdown and treatment with TGF- $\beta 1$, suggesting that ARK5 may reduce invasion and migration in HCC cells. Furthermore, these data also indicated that the treatment of Huh7 cells with TGF- $\beta 1$ resulted in decreased E-cadherin expression and increased expression of vimentin compared with the control. However, the downregulation of ARK5 was able to reverse TGF- $\beta 1$-induced EMT in Huh7 cells. These results indicated that the expression of ARK5 may serve an important role in cellular invasion and metastasis and EMT phenotype changes of HCC cells.

In summary, the present study demonstrated that inhibiting the expression of ARK5 may reduce the ability of the HCC cells to invade and metastasize via EMT. Therefore, ARK5 has the potential to be a molecular target for the development of novel therapy of HCC, which focuses on cell invasion.

\section{References}

1. Waly Raphael S, Yangde Z and Yuxiang C: Hepatocellular carcinoma: Focus on different aspects of management. ISRN Oncol 2012: 421673, 2012

2. Siegel RL, Miller KD and Jemal A: Cancer statistic, 2015. CA Cancer J Clin 65: 5-29, 2015.

3. Thiery JP and Sleeman JP: Complex networks orchestrate epithelial-mesenchymal transitions. Nat Rev Mol Cell Biol 7: 131-142, 2006.

4. Thiery JP, Acloque H, Huang RY and Nieto MA Epithelial-mesenchymal transitions in development and disease. Cell 139: 871-890, 2009.

5. Kaufhold S and Bonavida B: Central role of Snaill in the regulation of EMT and resistance in cancer: A target for therapeutic intervention. J Exp Clin Cancer Res 33: 62, 2014.

6. Christiansen JJ and Rajasekaran AK: Reassessing epithelial to mesenchymal transition as a prerequisite for carcinoma invasion and metastasis. Cancer Res 66: 8319-8326, 2006.

7. Lee TK, Poon RT, Yuen AP, Ling MT, Kwok WK, Wang XH, Wong YC, Guan XY, Man K, Chau KL and Fan ST: Twist overexpression correlates with hepatocellular carcinoma metastasis through induction of epithelialmesenchymal transition. Clin Cancer Res 12: 5369-5376, 2006.

8. Suzuki A, Kusakai G, Kishimoto A, Lu J, Ogura T, Lavin MF and Esumi H: IdentiWcation of a novel protein kinase mediating Akt survival signaling to the ATM protein. J Biol Chem 278: 48-53, 2003.

9. Kusakai G, Suzuki A, Ogura T, Miyamoto S, Ochiai A, Kaminishi $\mathrm{M}$ and Esumi H: ARK5 expression in colorectal cancer and its implications for tumor progression. Am J Pathol 164: 987-995, 2004.

10. Ekstrand AI, Jonsson M, Lindblom A, Borg A and Nilbert M: Frequent alterations of the PI3K/AKT/mTOR pathways in hereditary nonpolyposis colorectal cancer. Fam Cancer 9: 125-129, 2010 .

11. Li B, Tsao SW, Li YY, Wang X, Ling MT, Wong YC, He QY and Cheung AL: Id-1 promotes tumorigenicity and metastasis of human esophageal cancer cells through activation of PI3K/AKT signaling pathway. Int J Cancer 125: 2576-2585, 2009.

12. Ohta T, Isobe M, Takahashi T, Saitoh-Sekiguchi M, Motoyama T and Kurachi H: The Akt and ERK activation by platinum-based chemotherapy in ovarian cancer is associated with favorable patient outcome. Anticancer Res 29: 4639-4647, 2009.

13. Simon PO Jr, McDunn JE, Kashiwagi H, Chang K, Goedegebuure PS, Hotchkiss RS and Hawkins WG: Targeting AKT and with the proapoptotic peptide, TAT-CTMP: A novel strategy for the treatment of human pancreatic adenocarcinoma. Int J Cancer 125: 942-951, 2009.

14. Chang XZ, Yu J, Liu HY, Dong RH and Cao XC: ARK5 is associated with the invasive and metastatic potential of human breast cancercells. J Cancer Res Clin Oncol 138: 247-254, 2012.

15. Lu S, Niu N, Guo H, Tang J, Guo W, Liu Z, Shi L, Sun T, Zhou F, Li H, Zhang J and Zhang B: ARK5 promotes glioma cell invasion, andits elevated expression is correlated with poorclinical outcome. Eur J Cancer 49: 752-763, 2013.

16. Bustin SA: Absolute quantification of mRNA using real-time reverse transcription polymerase chain reaction assays. J Mol Endocrinol 25: 169-193, 2000.

17. Zheng X, Carstens JL, Kim J, Scheible M, Kaye J, Sugimoto H, Wu CC, LeBleu VS and Kalluri R: Epithelialto-mesenchymal transition is dispensable for metastasis but induces chemoresistance in pancreatic cancer. Nature 527: 525-530, 2015.

18. Liu Y, DU F, Zhao Q, Jin J, Ma X and Li H: Acquisition of 5-fluorouracil resistanceinduces epithelial-mesenchymal transitions through the Hedgehog signaling pathway in HCT-8 colon cancer cells. Oncol Lett 9: 2675-2679, 2015.

19. Fischer KR, Durrans A, Lee S, Sheng J, Li F, Wong ST, Choi H, El Rayes T, Ryu S, Troeger J, et al: Epithelial-to-mesenchymal transition is not required for lung metastasis but contributes tochemoresistance. Natur 527: 472-476, 2015.

20. Mallini P, Lennard T, Kirby J and Meeson A: Epithelial-to-mesenchymal transition: What is the impact on breast cancer stem cells and drug resistance. Cancer Treat Rev 40: 341-348, 2014

21. Xu T, Zhang J, Chen W, Pan S, Zhi X, Wen L, Zhou Y, Chen BW, Qiu J, Zhang Y, et al: ARK5 promotes doxorubicin resistance in hepatocellular carcinoma via epithelial-mesenchymal transition. Cancer Lett 377: 140-148, 2016. 
22. Zhang HY, Li JH, Li G and Wang SR: Activation of ARK5/miR-1181/HOXA10 axis promotes epithelial-mesenchymal transition in ovarian cancer. Oncol Rep 34: 1193-1202, 2015.

23. Li M, Zheng $\mathrm{C}, \mathrm{Xu} \mathrm{H}, \mathrm{He} \mathrm{W}$, Ruan Y, Ma J, Zheng J, Ye C and Li W: Inhibition of AMPK-related kinase 5 (ARK5) enhances cisplatin cytotoxicity in non-small cell lung cancer cells through regulation of epithelial-mesenchymal transition. Am J Trans Res 9: 1708-1719, 2017.

24. Park NR, Cha JH, Jang JW, Bae SH, Jang B, Kim JH, Hur W, Choi JY and Yoon SK: Synergistic effects of CD44 and TGF- $\beta 1$ through AKT/GSK-3 $\beta / \beta$-catenin signaling during epithelial-mesenchymal transition in liver cancer cells. Biochem Biophys Res Commun 477: 568-574, 2016.

25. El-Serag HB: Hepatocellular carcinoma. N Engl J Med 365: 1118-1127, 2011.

26. Kanda M, Sugimoto H and Kodera Y: Genetic and epigenetic aspects of initiation and progression of hepatocellular carcinoma. World J Gastroenterol 21: 10584-10597, 2015.

27. Kawasaki K, Smith RS Jr, Hsieh CM, Sun J, Chao J and Liao JK: Activation of and the phosphatidylinositol 3-kinase/protein kinase Akt pathway mediates nitric oxide-induced endothelial cell migration and angiogenesis. Mol Cell Biol 23: 5726-5737, 2003.

28. Li G, Satyamoorthy K and Herlyn M: N-cadherin-mediated intercellular interactions promote survival and migration of melanoma cells. CancerRes 61: 3819-3825, 2001.

29. Liu W, Liu Y and Lowe WL Jr: The role of phosphatidylinositol 3-kinaseand the mitogen-activated protein kinases in insulin-like growth factor-I-mediated effects in vascular endothelial cells. Endocrinology 142: 1710-1719, 2001.

30. Choi SS and Diehl AM: Epithelial-to-mesenchymal transitions in the liver. Hepatology 50: 2007-2013, 2009.
31. Xia H, Ooi LL and Hui KM: MicroRNA-216a/217-induced epithelial-mesenchymal transition targets PTEN and SMAD7 to promote drug resistance and recurrence of liver cancer. Hepatology 58: 629-641, 2013.

32. Thomson S, Petti F, Sujka-Kwok I, Mercado P, Bean J, Monaghan M, Seymour SL, Argast GM, Epstein DM and Haley JD: A systems view of epithelial-mesenchymal transition signaling states. Clin Exp Metastasis 28: 137-155, 2011.

33. Chen D, Liu G, Xu N, You X, Zhou H, Zhao X and Liu Q: Knockdown of ARK5 expression suppresses invasion and metastasis of gastric cancer. Cell Physiol Biochem 42: 1025-1036, 2017.

34. Chen XF, Zhang HJ, Wang HB, Zhu J, Zhou WY, Zhang H, Zhao MC, Su JM, Gao W, Zhang L, et al: Transforming growth factor- $\beta 1$ induces epithelial-to mesenchymal transition in human lung cancer cells via PI3K/Akt and MEK/Erk1/2 signaling pathways. Mol Biol Rep 39: 3549-3556, 2012.

35. Xu Z, Shen MX, Ma DZ, Wang LY and Zha XL: TGF-beta1-promoted epithelial-to-mesenchymal transformation and cell adhesion contribute to TGF-beta1-enhanced cell migration in SMMC-7721 cells. Cell Res 13: 343-350, 2003.

36. Dang H, Ding W, Emerson D and Rountree CB: Snail1 induces epithelial-tomesenchymal transition and tumor initiating stem cell characteristics. BMC Cancer 11: 396, 2011.

37. Ji J and WangXW: Clinical implications of cancer stem cell biology in hepatocellular carcinoma. Semin Oncol 39: 461-472, 2012.

38. Yang JD, Nakamura I and Roberts LR: The tumor microenvironment in hepatocellular carcinoma: Current status and therapeutic targets. Semin Cancer Biol 21: 35-43, 2011.

This work is licensed under a Creative Commons Attribution-NonCommercial-NoDerivatives 4.0 International (CC BY-NC-ND 4.0) License. 